\title{
REPRESENTATIONS OF FUNCTIONS BY DERIVATIVES BY
}

\author{
S. J. AGRONSKY, R. BISKNER, A. M. BRUCKNER ${ }^{1}$ AND J. MAŔ'IK
}

\begin{abstract}
Let $\Delta^{\prime}$ be the class of all derivatives. The main goal of this paper is the investigation of the vector space generated by $\Delta^{\prime}$ and $O^{\prime}$ Malley's class $B_{1}^{*}$; this space is identical with our system $\left[\Delta^{\prime}\right]$. We show, in particular, that each approximately continuous function and each approximate derivative belongs to $\left[\Delta^{\prime}\right]$ and that $\left[\Delta^{\prime}\right]$ is the system of all functions of the form $g^{\prime}+h k^{\prime}$, where $g, h$ and $k$ are differentiable.
\end{abstract}

1: Introduction. In recent years a number of authors have studied various sorts of functions important to differentiation theory and found that in many instances their functions possess structures very similar to the structure of ordinary derivatives. For example, each approximately differentiable function is differentiable on a dense, open set and its approximate derivative has the Darboux property, belongs to the first class of Baire and even to Zahorski's class $\Re_{3}$, Weil's class $Z$ and Preiss' class $\mathfrak{K}_{3}^{*}$. (See [5], [13], [11], [12], [10].) Related results involving Peano derivatives, selective derivatives, approximately continuous functions and $B_{1}^{*}$ functions are also known ([11], [12], [6], [7]).

The purpose of this paper is to point out some other relations between functions of certain of the classes mentioned above and the derivatives. In Theorem 3, for example, we show that each approximate derivative, each approximately continuous function and each $B_{1}^{*}$ function can be expressed in the form $g^{\prime}+h k^{\prime}$ with $g, h$ and $k$ differentiable. This result is a consequence of Theorem 2 which characterizes a certain class of functions in three ways. We then study this class and some related classes in detail. In particular, we investigate functions representable in the form $h k^{\prime}$ with $k$ differentiable and $h$ either differentiable or continuous or a derivative. Our results show, among other things, that the function $g^{\prime}$ in the representation $g^{\prime}+h k^{\prime}$ cannot be dropped in Theorem 2 .

The work presented here originated with a paper by the first three authors submitted to the Transactions of the American Mathematical Society in June of 1978, but never published, in which they showed that approximate derivatives, approximately continuous functions and $B_{1}^{*}$ functions can be written as $f_{1}+f_{2} f_{3} f_{4}$ $+f_{5} f_{6} f_{7}$ where each of these seven functions is a derivative. After reading a preprint of that paper the fourth author, with extensive collaboration from the third, shortened the proofs and improved the results to the present form.

Received by the editors June 26, 1978 and, in revised form, September 29, 1978 and February 5, 1980. 1980 Mathematics Subject Classification. Primary 26A24; Secondary 26A21, 26A27.

Key words and phrases. Derivatives, approximate derivatives, approximately continuous functions, functions of Baire class 1 .

${ }^{1}$ This author's work was supported in part by a grant from the National Science Foundation. 
2. Preliminaries. The main results of this paper depend on certain concepts, constructions of functions and decompositions of sets with special properties. We find it convenient to set off these technical considerations in this preliminary section.

We begin with some notation. Throughout this paper, $R=(-\infty, \infty)$; the word function means a mapping to $R ; J$ is the set of all integers; if $V$ is either an open set in $R$ or an interval (not necessarily open), then $\Delta(V)$ is the family of all functions differentiable on $V$ and $\Delta^{\prime}(V)=\left\{f^{\prime} ; f \in \Delta(V)\right\}$; we set $\Delta=\Delta(R)$, $\Delta^{\prime}=\Delta^{\prime}(R)$ and $\mathfrak{D}=\left\{g \mid S ; g \in \Delta^{\prime}, S \subset R\right\}$. For each open interval $I$ let $\mathfrak{A}(I)$ be the system of all increasing functions $\lambda$ on $J$ such that inf $\lambda(J)=\inf I$, sup $\lambda(J)=$ sup $I$. If $\lambda \in \mathfrak{A}(I)$ and $j \in J$, we write $\lambda_{j}=\lambda(j)$. If $I$ is an open interval, $f$ and $w$ functions continuous on $I$ and if $w>0$ on $I$, then $\mathfrak{A}(I, f, w)$ is the system of all $\lambda \in \mathfrak{U}(I)$ with the following property: if $j \in J$ and if $x, y, z \in\left[\lambda_{j-1}, \lambda_{j}\right]$, then $|f(y)-f(x)|<w(z)$. If $f$ and $g$ are mappings, then $f \circ g$ is the mapping $h$ defined by $h(x)=f(g(x))$ for all $x$ for which the expression $f(g(x))$ has a meaning.

Lemma 1. Let $I, f$ and $w$ be as above. Then $\mathfrak{U}(I, f, w) \neq \varnothing$.

(The proof is left to the reader.)

Lemma 2. Let $Q>1$, let I be an open interval and let $\varphi, w$ be functions continuous on $I, w>0$ on I. Then there are functions $v, h$ and $k$ infinitely differentiable on $I$ with the following properties:

(1) $|\varphi-v|<w^{2}, v^{\prime}=h k^{\prime}, 0 \leqslant h<w, 0 \leqslant k<w$ on $I$.

(2) For each $x \in I$ there is a $\theta \in[0, Q]$ and points $x_{1}, x_{2} \in I$ such that $x_{1} \leqslant x<$ $x_{2}$ and $v^{\prime}(x)=\theta\left(\varphi\left(x_{2}\right)-\varphi\left(x_{1}\right)\right) /\left(x_{2}-x_{1}\right)$.

Proof. There is an $\varepsilon \in\left(0, \frac{1}{4}\right)$ and nonnegative functions $\alpha, \beta, \gamma_{-1}, \gamma_{1}$ infinitely differentiable on $R$ with the following properties: $\alpha=0$ on $(-\infty, 2 \varepsilon), \alpha=1$ on $(1-2 \varepsilon, \infty), 0 \leqslant \alpha^{\prime} \leqslant Q$ on $R, \beta=0$ on $(-\infty, \varepsilon) \cup(1-\varepsilon, \infty), \beta=1$ on $(2 \varepsilon, 1-$ $2 \varepsilon), 0 \leqslant \beta \leqslant 1$ on $R, \gamma_{-1}=0$ on $(-\infty, 0), \gamma_{-1}=1-\alpha$ on $(\varepsilon, \infty), 0 \leqslant \gamma_{-1} \leqslant 1$ on $R, \gamma_{1}=\alpha$ on $(-\infty, 1-\varepsilon), \gamma_{1}=0$ on $(1, \infty), 0 \leqslant \gamma_{1} \leqslant 1$ on $R$. Obviously, for $n=1$ and $-1, \alpha^{\prime}=n \beta \gamma_{n}^{\prime}$ on $R$. Let $\lambda \in \mathfrak{A}\left(I, \varphi, w^{2}\right)$. Define $\gamma_{0}=0$ on $R$. For $x \in R$ let $\tau_{j}(x)=\left(x-\lambda_{j-1}\right) /\left(\lambda_{j}-\lambda_{j-1}\right)$. For $j \in J$ let $\eta_{j}=\varphi\left(\lambda_{j}\right)-\varphi\left(\lambda_{j-1}\right), \sigma_{j}=$ $\operatorname{sgn} \eta_{j}, \quad v=\varphi\left(\lambda_{j-1}\right)+\eta_{j} \cdot \alpha \circ \tau_{j}, \quad h=\left|\eta_{j}\right|^{1 / 2} \cdot \beta \circ \tau_{j}$ and $k=\left|\eta_{j}\right|^{1 / 2} \cdot \gamma_{\sigma_{j}} \circ \tau_{j}$ on $\left[\lambda_{j-1}, \lambda_{j}\right]$. It is easy to see that the functions $v, h$ and $k$ satisfy our requirements.

Proposition 1. Let $Q>1$. Let $S$ be a closed set in $R, T=R \backslash S$. Let $F \in \Delta$ and $G \in \Delta(T)$. Then there are $g, h, k \in \Delta$ with the following properties: $h \geqslant 0, k \geqslant 0$ on $R ; h, k$ are infinitely differentiable on $T ; h=k=0$ on $S ; g=F$ and $g^{\prime}=g^{\prime}+h k^{\prime}$ $=F^{\prime}$ on $S ; g^{\prime}+h k^{\prime}=G^{\prime}$ on $T$; if $I$ is a component of $T$ and if $x \in I$, then there is $a \xi \in I$ and $a \theta \in[0, Q]$ such that $\left(h k^{\prime}\right)(x)=\theta \cdot(G-F)^{\prime}(\xi)$.

Proof. Let $w \in \Delta, w=0$ on $S, w>0$ on $T$. Define $\varphi=G-F$ on $T$. On each component $I$ of $T$ construct $v, h$ and $k$ according to Lemma 2. Set $g_{0}=\varphi-v$ on $T, g_{0}=h=k=0$ on $S$ and $g=g_{0}+F$ on $R$. Obviously $g_{0}^{\prime}=h^{\prime}=k^{\prime}=0$ on $S$ and $g^{\prime}+h k^{\prime}=g^{\prime}=F^{\prime}$ on $S$. Since $g+v=\varphi+F=G$ on $T$, we have $g^{\prime}+h k^{\prime}$ $=G^{\prime}$ on $T$. The rest follows from Lemma 2 . 
Lemma 3. Let $A$ be closed in $R$ with int $A=\varnothing$ and let $B$ be open in $R$. Then there is a countable system of pairwise disjoint closed sets with union $A \cap B$.

Proof. We may suppose that $B$ is an interval. Since int $A=\varnothing$, there is a $\lambda \in \mathfrak{A}(B)$ such that $A \cap \lambda(J)=\varnothing$. The system $\left\{A \cap\left[\lambda_{j-1}, \lambda_{j}\right] ; j \in J\right\}$ has the required properties.

REMARK. If we add to the assumptions of Lemma 4 the requirement that the sets $A_{n}$ be pairwise disjoint, we obtain a part of Theorem 4.5 of Petruska-Laczkovich [9].

Lemma 4. Let $A, A_{1}, A_{2}, \ldots$ be closed, $A=\cup A_{n} \subset R$, int $A=\varnothing$. Let $f$ be $a$ function such that $f \mid A_{n} \in \mathfrak{D}$ for $n=1,2, \ldots$ Then $f \mid A \in \mathfrak{D}$.

Proof. By Lemma 3, for each natural number $n$ there is a countable system $\mathscr{B}_{n}$ of pairwise disjoint closed sets with union $A_{n} \backslash\left(A_{1} \cup \cdots \cup A_{n-1}\right)$. The union $\mathscr{B}$ of all the systems $\mathscr{B}_{n}$ is a countable system of pairwise disjoint closed sets with union $A$ and we have $f \mid B \in \mathfrak{D}$ for each $B \in \mathfrak{B}$. Now we apply the theorem of PetruskaLaczkovich mentioned above.

LemMA 5. Let $\mathscr{K}$ be a system of subsets of $R$ such that $A \in \mathscr{K}$ whenever $A \subset B \in \mathscr{K}$. Then the following two conditions are equivalent:

(i) There is a countable system of closed elements of $\mathcal{H}$ with union $R$.

(ii) For each nonempty closed set $S \subset R$ there is an open interval I such that $\varnothing \neq S \cap I \in \mathcal{K}$.

Proof. The condition (ii) follows from (i) by Baire's category theorem. Now suppose that (ii) holds. Let $\mathfrak{T}$ be the system of the unions of all countable systems of closed elements of $\mathcal{K}$. If $A$ is an $F_{\sigma}$-set, $A \subset M \in \mathfrak{N}$, then, obviously, $A \in \mathfrak{N}$. Let $T$ be the set of all points $x \in R$ for which there is an open interval $I$ such that $x \in I \in \mathfrak{N}$. Since for each $x \in T$ there is such an interval with rational endpoints, we have $T \in \mathfrak{N}$. Set $S=R \backslash T$ and suppose that $S \neq \varnothing$. There is an open interval $I$ such that $\varnothing \neq S \cap I \in \mathcal{K}$. It follows that $I=(S \cap I) \cup(T \cap I) \in$ $\mathfrak{R}, I \subset T$, which is a contradiction. Therefore, $S=\varnothing$ and $R=T \in \mathfrak{R}$.

We shall need a bit more notation.

Let $\mathfrak{F}$ be the system of all (finite real) functions on $R$. Let $C, C_{a p}, B_{1}, \Delta_{a p}, \Delta_{a p}^{\prime}$ be the systems of all continuous functions, of all approximately continuous functions, of all functions of Baire class 1, of all approximately differentiable functions and of all approximate derivatives on $R$, respectively. For each system $\mathfrak{B} \subset \mathfrak{F}$ let $b \mathfrak{B}$ be the system of all bounded elements of $\mathfrak{B}$ and let $[\mathfrak{B}]$ be the system of all functions $f$ on $R$ with the following property: There are $v_{n} \in \mathfrak{B}$ and closed sets $A_{n}$ such that $\cup A_{n}=R$ and $f=v_{n}$ on $A_{n}(n=1,2, \ldots)$.

REMARK. Obviously $\mathfrak{B} \subset[\mathfrak{B}]=[[\mathfrak{B}]]$. If $\mathfrak{B}$ is a vector space (an algebra), then [ $\mathfrak{B}]$ is a vector space (an algebra). It is well known that a function $f$ is in $B_{1}$ if and only if $\{x ; f(x)>c\}$ and $\{x ; f(x)<c\}$ are $F_{\sigma}$-sets for each $c \in R$. This easily implies that $\left[B_{1}\right]=B_{1}$.

We shall of ten apply the fact that for each $f \in B_{1}$ and each closed set $S \neq \varnothing$ there is an $x \in S$ such that $f \mid S$ is continuous at $x$. 
Proposition 2. Let $\mathfrak{B} \subset \mathfrak{F}$ and $f \in \mathfrak{F}$. Then $f \in[\mathfrak{B}]$ if and only if for each closed set $S \neq \varnothing$ there is an open interval $I$ and $a v \in \mathfrak{B}$ such that $S \cap I \neq \varnothing$ and $f=v$ on $S \cap I$.

(This follows from Lemma 5.)

Proposition 3. Let $f \in B_{1}$ and let the range of $f$ be isolated. Then $f \in[C]$.

Proof. Let $S$ be closed, $S \neq \varnothing$. There is an open interval $I$ such that $S \cap I \neq \varnothing$ and that $f$ is constant on $S \cap I$. Now we apply Proposition 2 .

TheORem 1. Let $f \in B_{1}$. Then there are $f_{n} \in[C](n=1,2, \ldots)$ such that $f_{n} \rightarrow f$ uniformly.

Proof. For $n=1,2, \ldots$ there is an $f_{n} \in B_{1}$ such that $f_{n}(R)$ is isolated and that $\left|f-f_{n}\right|<1 / n$ on $R$. By Proposition 3 we have $f_{n} \in[C]$.

Proposition 4. Let $f \in\left[\Delta^{\prime}\right]$ and let $S$ be a nowhere dense set. Then $f \mid S \in \mathfrak{D}$.

(This follows from Lemma 4.)

3. The representation $f=g^{\prime}+h k^{\prime}$. We are now ready to give three characterizations of the class $\left[\Delta^{\prime}\right]$. (See Theorem 2.) We prove, in particular, that every function $f \in\left[\Delta^{\prime}\right]$ can be expressed in the form $f=g^{\prime}+h k^{\prime}$ where $g, h$ and $k$ are differentiable. Theorem 3 shows that $\left[\Delta^{\prime}\right]$ contains all approximately continuous functions, all approximate derivatives and all functions of O'Malley's class $B_{1}^{*}$.

THEOREM 2. Let $f \in \mathfrak{F}$. Then the following four conditions are equivalent:

(i) There are $g$, $h$ and $k$ in $\Delta$ such that $h^{\prime}, k^{\prime} \in[C]$ and $f=g^{\prime}+h k^{\prime}$.

(ii) There is $a \varphi \in \Delta^{\prime}$ and $a \psi \in[C]$ such that $f=\varphi+\psi$.

(iii) $f \in\left[\Delta^{\prime}\right]$.

(iv) There is a dense open set $T$ such that $f \mid T \in \Delta^{\prime}(T)$ and $f \mid R \backslash T \in \mathfrak{D}$.

Proof. The implications (i) $\rightarrow$ (ii) and (ii) $\rightarrow$ (iii) are obvious. Now let $f \in\left[\Delta^{\prime}\right]$ and let $T$ be the set of all points $x \in R$ for which there is an open interval $I$ containing $x$ such that $f \mid I \in \mathfrak{D}$. Then $T$ is open and, by Proposition 2 , dense. It is easy to see that $f \mid T \in \Delta^{\prime}(T)$ and it follows from Proposition 4 that $f \mid R \backslash T \in \mathfrak{D}$. Thus, (iii) $\rightarrow$ (iv). Finally, the implication (iv) $\rightarrow$ (i) follows directly from Proposition 1.

COROLlaRY 1. The system $\left[\Delta^{\prime}\right]$ is the vector space generated by $\Delta^{\prime}$ and $[C]$.

Remark. It follows from Theorem 2 that every function $f \in\left[\Delta^{\prime}\right]$ can be expressed in the form $g^{\prime}+h k^{\prime}$ with $g, h$ and $k$ in $\Delta$. If either of the functions $h^{\prime}$ or $k^{\prime}$ is locally summable, then $h k^{\prime} \in \Delta^{\prime}$ and, of course, $f \in \Delta^{\prime}$ as well. (See, e.g., Fleissner [4] for a discussion of such products $h k^{\prime}$.) Condition (iv) of Theorem 2 implies that, for example, the function sgn is in $\left[\Delta^{\prime}\right]$ so that sgn $=g^{\prime}+h k^{\prime}$ for some $g, h$ and $k$ in $\Delta$; but, no matter how we choose such $g, h$ and $k$, neither $h^{\prime}$ nor $k^{\prime}$ is locally summable. (We see that the product of a differentiable function and a derivative need not be in $\Delta^{\prime}$; however, according to Theorem 4 of the next section, such a product is always in $\left[\Delta^{\prime}\right]$.) 
Theorem 3 below describes three important classes of functions contained in $\left[\Delta^{\prime}\right]$.

THEOREM 3. Each approximately continuous function, each approximate derivative and each function in $B_{1}^{*}$ meets the conditions of Theorem 2.

Proof. According to Lemma 5, the class $B_{1}^{*}$ defined in [6] is the same as our class $[C]$ and, obviously, $[C] \subset\left[\Delta^{\prime}\right]$. In [8], O'Malley proved that each approximate derivative is in $\left[\Delta^{\prime}\right]$. Finally, let $f$ be approximately continuous and let $S$ be a nonempty closed set. Since $f \in B_{1}$, there exists an open interval $I$ and an $M \in R$ such that $S \cap I \neq \varnothing$ and that $|f| \leqslant M$ on $S \cap I$. Let $\varphi=\max (-M, \min (f, M))$. Since $\varphi$ is approximately continuous and bounded, it is the derivative of its indefinite Lebesgue integral. This implies that $f \mid S \cap I \in \mathfrak{D}$. According to Lemma 5 we have $f \in\left[\Delta^{\prime}\right]$.

REMARK. We close this section with the observation that each function in the first class of Baire can be approximated uniformly by a function of the form $g^{\prime}+h k^{\prime}$ with $g, h$ and $k$ in $\Delta$. This follows immediately from Theorems 1 and 2 .

4. The use of bounded derivatives. The definition of $\left[\Delta^{\prime}\right]$ involves a certain decomposition of its members into derivatives which must be finite but need not be bounded. In this section we show that we could have restricted our attention to bounded derivatives and we obtain some additional results. For example, Corollary 3 shows that $\left[\Delta^{\prime}\right]$ is precisely the class of functions representable as $g^{\prime}+h k^{\prime}$ with $g$, $h$ and $k$ in $\Delta$.

Lemma 6. Let $L$ be an interval and $Q$ a compact subset of $L$. Let $f \in \Delta(L)$ and $\beta \in R$. Suppcse that $|f(t)-f(x)| \leqslant \beta|t-x|$, whenever $x \in Q$ and $t \in L$. Then there is a $g \in \Delta(L)$ such that $\left|g^{\prime}\right| \leqslant \beta+1$ on $L$ and $g^{\prime}=f^{\prime}$ on $Q$.

Proof. We may suppose that $Q$ has at least two points and that $L$ is the smallest interval containing $Q$. Let $S=(a, b)$ be a component of $L \backslash Q$. According to [9, Lemma 4.15], there is a function $g_{s} \in \Delta([a, b])$ such that $g_{s}^{\prime}=f^{\prime}$ on $\{a, b\}$, $\left|g_{S}^{\prime}\right| \leqslant \beta+1$ on $[a, b]$ and that the graph of $g_{S}$ is contained in the convex hull of the graph of $f \mid[a, b]$. Now we set $g=f$ on $Q$ and $g=g_{S}$ on each component $S$ of $L \backslash Q$. It is easy to see that $g$ has the required properties.

Proposition 5. We have $\Delta^{\prime} \subset\left[b \Delta^{\prime}\right]$.

Proof. Let $f \in \Delta$. Let $A_{n}$ be the set of all points $x \in R$ such that $|f(t)-f(x)| \leqslant$ $n|t-x|$ for all $t \in R$ for which $|t-x|<1 / n(n=1,2, \ldots)$. It is easy to see that $A_{n}$ is closed and that $\cup A_{n}=R$. Fix an $n$, choose a $\delta \in(0,1 / n)$ and for $j \in J$ define $L_{j}=[(j-1) \delta, j \delta]$. On each interval $L_{j}$ construct a function $g_{j}=g$ according to Lemma 6 where we take $Q=A_{n} \cap L_{j}$ and $\beta=n$. If $x$ is either $(j-1) \delta$ or $j \delta$ and if $x \notin Q$, we choose $g_{j}$ in such a way that $g_{j}^{\prime}(x)=0$. There are numbers $c_{j}$ such that $g_{j}(j \delta)+c_{j}=g_{j+1}(j \delta)+c_{j+1}$. Setting $h=g_{j}+c_{j}$ on $[(j-1) \delta, j \delta]$ for $j \in J$ we have $\left|h^{\prime}\right| \leqslant n+1$ on $R$ and $h^{\prime}=f^{\prime}$ on $A_{n}$. This completes the proof.

LemMa 7. Let $f \in B_{1}$. Then there is a countable system $\mathcal{Q}$ of closed sets with union $R$ such that $f \mid A$ is bounded for each $A \in \mathbb{Q}$. 
(This follows from Lemma 5.)

Proposition 6. We have $C_{a p} \subset\left[b C_{a p}\right]$.

(This follows easily from Lemma 7.)

Corollary 2. We have $\left[C_{a p}\right]=\left[b C_{a p}\right],\left[\Delta^{\prime}\right]=\left[b \Delta^{\prime}\right]$.

(This follows from Propositions 5 and 6.)

THEOREM 4. Let $\varphi \in\left[C_{a p}\right], \psi \in\left[\Delta^{\prime}\right]$. Then $\varphi \psi \in\left[\Delta^{\prime}\right]$.

PROoF. It follows from Corollary 2 that there are $\varphi_{n} \in b C_{a p}, \psi_{n} \in b \Delta^{\prime}$ and closed sets $A_{n}$ such that $\cup A_{n}=R$ and $\varphi_{n} \psi_{n}=\varphi \psi$ on $A_{n}(n=1,2, \ldots)$. According to Theorem 3.6 of [4] we have $\varphi_{n} \psi_{n} \in \Delta^{\prime}$ for each $n$.

Corollary 3. Let $f \in \mathfrak{F}$. Then $f \in\left[\Delta^{\prime}\right]$ if and only if there are $g, h$ and $k$ in $\Delta$ such that $f=g^{\prime}+h k^{\prime}$.

(This follows from Theorems 2 and 4.)

Corollary 4. Let $\Delta \subset \mathfrak{B} \subset\left[C_{a p}\right], \Delta \subset \mathfrak{W} \subset \Delta_{a p}$. Then $\left[\Delta^{\prime}\right]$ is the vector space generated by the products $h k_{a p}^{\prime}$, where $h \in \mathfrak{B}$ and $k \in \mathfrak{B}$.

(This follows from Theorems 2-4.)

REMARK. We see, in particular, that $h k_{a p}^{\prime} \in\left[\Delta^{\prime}\right]$ whenever $h$ and $k$ are in $\Delta_{a p}$. It is also clear that two parts of Theorem 3 follow immediately from Theorem 4 . We cannot, however, extend Theorem 4 to the assertion that $\varphi \psi \in\left[\Delta^{\prime}\right]$ whenever $\varphi$ and $\psi$ are in $\left[\Delta^{\prime}\right]$. In fact, it is not difficult to construct a $\varphi \in b \Delta^{\prime}$ such that $\varphi^{2}$ is not a derivative on any interval (see [2]). It is clear that $\varphi^{2}$ cannot be in [ $\left.\Delta^{\prime}\right]$.

5. Representations of the form $h k^{\prime}$. It is natural to ask whether the function $g^{\prime}$ in the representation $f=g^{\prime}+h k^{\prime}$ (see Corollary 3 ) is really necessary, i.e. whether one can represent each function in $\left[\Delta^{\prime}\right]$ as $h k^{\prime}$ with $h$ and $k$ in $\Delta$. This turns out not to be possible even for the function sgn; namely, according to a remark following Theorem 7.5 of [3], the product of a continuous function with a derivative always has the Darboux property. In fact, we shall see that even "very well behaved" Darboux functions may fail to be representable in that form.

In this section we shall consider functions of the form $h k^{\prime}$ with $k \in \Delta$ and $h$ in various classes. Let $\mathfrak{P}_{0}=\left\{h k^{\prime} ; h, k \in \Delta\right\}, \mathfrak{P}_{1}=\left\{h k^{\prime} ; h \in C, k \in \Delta\right\}, \mathfrak{P}_{2}=\left\{h^{\prime} k^{\prime} ;\right.$ $h, k \in \Delta\}$. We begin with an elementary lemma.

Lemma 8. Let $a, b, c \in R, b<a \leqslant 0, c \neq 0$. Let $f(x)=x^{a} \sin \left(c x^{b}\right), g(x)=$ $x^{a} \cos \left(c x^{b}\right), h(x)=x^{a} \cdot\left(\sin \left(c x^{b}\right)\right)^{2}$ for $x>0$ and let $f=g=h=0$ on $(-\infty, 0]$. Then $f, g \in \Delta^{\prime}, h \notin \Delta^{\prime}$.

Proof. Set $F(x)=x^{a-b+1} \cdot \cos \left(c x^{b}\right), f_{0}(x)=(a-b+1) x^{a-b} \cdot \cos \left(c x^{b}\right), h_{1}(x)$ $=x^{a}, h_{2}(x)=x^{a} \cos \left(2 c x^{b}\right)$ for $x>0, F=f_{0}=h_{1}=h_{2}=0$ on $(-\infty, 0]$. As $F^{\prime}=f_{0}-b c f$, we have $f \in \Delta^{\prime}$; similarly it can be shown that $g \in \Delta^{\prime}$. Since $h_{1} \notin \Delta^{\prime}$, $h_{2} \in \Delta^{\prime}$ and $2 h=h_{1}-h_{2}$, we have $h \notin \Delta^{\prime}$. 
THEOREM 5. We have proper inclusions

$$
\Delta^{\prime} \subset \mathfrak{P}_{0} \subset \mathfrak{B}_{1} \subset \mathfrak{P}_{2}, \quad \mathfrak{P}_{1} \subset\left[\Delta^{\prime}\right],
$$

but neither the inclusion $\mathfrak{P}_{2} \subset\left[\Delta^{\prime}\right]$ nor the inclusion $\left[\Delta^{\prime}\right] \subset \mathfrak{P}_{2}$ is valid.

Proof. It follows immediately from Theorem 4 that $\mathfrak{P}_{1} \subset\left[\Delta^{\prime}\right]$; the other inclusions in (*) are obvious.

To see that $\mathfrak{B}_{0} \backslash \Delta^{\prime} \neq \varnothing$, set $\varphi(x)=x^{2} \sin x^{-3}, \psi(x)=x^{-2} \sin x^{-3}$ for $x>0$ and $\varphi=\psi=0$ on $(-\infty, 0]$. Obviously $\varphi \in \Delta$. By Lemma 8 we have $\psi \in \Delta^{\prime}$ and $\varphi \psi \notin \Delta^{\prime}$.

To see that $\mathfrak{P}_{1} \backslash \mathfrak{B}_{0} \neq \varnothing$, set $f(x)=x^{1 / 2} \sin x^{-1}, g(x)=x^{-1 / 2} \sin x^{-1}$ for $x>0$ and $f=g=0$ on $(-\infty, 0]$. Obviously $f \in C$. By Lemma 8 we have $g \in \Delta^{\prime}$ and $f g \notin \Delta^{\prime}$. Now suppose that $h, k \in \Delta$ and that $f g=h k^{\prime}$. If, e.g., $h(0)>0$, then $k^{\prime} \geqslant 0$ on some neighborhood of 0 so that, according to Theorem 4.2 of [4], $h k^{\prime} \in \Delta^{\prime}$ which is a contradiction. Therefore, $h(0)=0$. There is an $M \in R$ such that $|h(x) / x|<M$ for each $x \in(0,1)$. Set $x_{n}=(n \pi)^{-1}(n=1,2, \ldots)$. We have

$$
\int_{x_{n+1}}^{x_{n}} f g=\int_{n \pi}^{(n+1) \pi} t^{-2}(\sin t)^{2} d t \geqslant \pi x_{n+1}^{2} / 2
$$

on the other hand,

$$
\begin{aligned}
x_{n}^{-2} \cdot \int_{x_{n+1}}^{x_{n}} f g & \leqslant M\left|k\left(x_{n}\right)-k\left(x_{n+1}\right)\right| / x_{n} \\
& =M\left|\frac{k\left(x_{n}\right)-k(0)}{x_{n}}-\frac{k\left(x_{n+1}\right)-k(0)}{x_{n+1}} \cdot \frac{x_{n+1}}{x_{n}}\right| \rightarrow 0
\end{aligned}
$$

which is impossible.

According to [2] there is an $f \in \Delta^{\prime}$ such that $f^{2}$ is not a derivative on any interval. Then $f^{2} \in \mathfrak{B}_{2}$ but (see Theorem 2, (iv)) $f^{2} \notin\left[\Delta^{\prime}\right]$.

In the example below we shall show more than that the inclusion $\left[\Delta^{\prime}\right] \subset \mathfrak{B}_{2}$ is not valid. This will complete the proof of Theorem 5 .

EXAMPLE. Let $d_{n+1}<a_{n}<b_{n}<c_{n}<d_{n}, a_{n} \rightarrow 0$; let the set $G=\cup\left(a_{n}, d_{n}\right)$ have density 0 at 0 . Define $L_{n}=\left(b_{n}, c_{n}\right), \alpha_{n}=\left(c_{n}-b_{n}\right)^{-1}(n=1,2, \ldots)$. There is a function $F$ on $R$ which is infinitely differentiable on $(0, \infty)$ such that $F=0$ on $R \backslash G$ and $F^{\prime}>\alpha_{n}^{2}$ on $L_{n}$. Set $f=F_{a p}^{\prime}$. Then $f$ is infinitely many times approximately differentiable. Suppose that $f=h^{\prime} k^{\prime}$ with $h, k \in \Delta$. If $h^{\prime}$ and $k^{\prime}$ are both positive on $L_{n}$, we have, for each $x \in L_{n}$, either $h^{\prime}(x)>\alpha_{n}$ or $k^{\prime}(x)>\alpha_{n}$ whence $h^{\prime}+k^{\prime}>\alpha_{n}$ on $L_{n}$. The other possibility is that both $h^{\prime}$ and $k^{\prime}$ are negative on $L_{n}$ in which case we have $h^{\prime}+k^{\prime}<-\alpha_{n}$ on $L_{n}$. Let $\varphi=h+k$. Then

$$
\left|\varphi\left(c_{n}\right)-\varphi\left(b_{n}\right)\right|=\left|\int_{b_{n}}^{c_{n}} \varphi^{\prime}\right|>\alpha_{n}\left(c_{n}-b_{n}\right)=1
$$

for each $n$. This is impossible because $\varphi$ is continuous.

We note that the function $f$ is in $C_{a p} \cap[C] \cap \Delta_{a p}^{\prime}$; it is very well behaved except at the origin, yet cannot be expressed as the product of two derivatives.

RemarK. Suppose that $f_{0}>0, f_{1} \in C, f_{2} \in \Delta, f_{0}=f_{1} f_{2}^{\prime}$. Assume without loss of generality that both $f_{1}$ and $f_{2}^{\prime}$ are positive. By Theorem 4.2 of [4] we have $f_{0} \in \Delta^{\prime}$. Thus, each positive function in $\mathfrak{B}_{1}$ is a derivative. 
In contrast, observe that the function $\varphi \psi$ in the proof of Theorem 5 belongs to $\mathfrak{B}_{0}$, is nonnegative, but is not a derivative.

In general, if $\Phi \in \mathfrak{B}_{0} \backslash \Delta^{\prime}$ and $\Phi \geqslant 0$, then $\Phi+1 \notin \mathfrak{P}_{1}$ (for otherwise $\Phi$ would have to be in $\Delta^{\prime}$ ). Thus, neither $\mathfrak{B}_{0}$ nor $\mathfrak{B}_{1}$ is a vector space.

6. Additional remarks. The work of the previous sections suggests certain other possibilities. First, we may ask for other classes of functions $f$ for which the representation $f=g^{\prime}+h k^{\prime}$ with $g, h, k \in \Delta$ is valid (which means $f \in\left[\Delta^{\prime}\right]$ ). Possible candidates are various generalized derivatives (e.g. Peano derivatives and selective derivatives) or functions related to differentiation theory (e.g. Darboux Baire 1 functions or semicontinuous functions). We do not know whether each Peano or selective derivative is in $\left[\Delta^{\prime}\right]$. But there exist semicontinuous Darboux functions which vanish almost everywhere and are positive on some dense set (see [1]). Such a function cannot be a derivative on any interval and therefore cannot belong to $\left[\Delta^{\prime}\right]$. It would be of interest to know which generalized derivatives are in $\left[\Delta^{\prime}\right]$.

We ended $\S 3$ with the observation that each Baire 1 function is a uniform limit of functions of the form $g^{\prime}+h k^{\prime}$ with $g, h, k \in \Delta$. It is, of course, clear that an $f \in B_{1}$ need not itself be expressible in this form. We have not been able to determine, however, whether each $f \in B_{1}$ is in the algebra generated by $\Delta^{\prime}$. More specifically, we have not been able to construct an $f \in B_{1}$ which cannot be expressed in the form $f=g^{\prime}+h^{\prime} k^{\prime}$ with $g, h, k \in \Delta$.

\section{REFERENCES}

1. S. Agronsky, Characterizations of certain subclasses of the Baire class 1, Doctoral Dissertation, Univ. of California, Santa Barbara, 1974.

2. A. Bruckner; Inflexible derivatives, Quart. J. Math. Oxford Ser. 29 (1978), 1-10.

3. A. M. Bruckner and J. G. Ceder, Darboux continuity, Jber. Deutsch. Math.-Verein. 67 (1964/65), Abt. 1, 93-117.

4. R. Fleissner, Multiplication and the fundamental theorem of calculus, Real Analysis Exchange 2 (1976), 7-39.

5. G. Goffman and C. J. Neugebauer, On approximate derivatives, Proc. Amer. Math. Soc. 11 (1960), 962-966.

6. R. J. O’Malley, Baire*1, Darboux functions, Proc. Amer. Math. Soc. 60 (1976), 187-192.

7. Selective derivatives, Acta Math. Acad. Sci. Hungar. 29 (1977), 77-97.

8. Decompositions of approximate derivatives, Proc. Amer. Math. Soc. 69 (1978), 243-247.

9. G. Petruska and M. Laczkovich, Baire 1 functions, approximately continuous functions and derivatives, Acta Math. Acad. Sci. Hungar. 25 (1974), 189-212.

10. D. Preiss, Level sets of derivatives, Trans. Amer. Math. Soc. (to appear).

11. C. Weil, On properties of derivatives, Trans. Amer. Math. Soc. 114 (1965), 363-376.

12. A property for certain derivatives, Indiana Univ. Math. J. $23(1973 / 74), 527-536$.

13. Z. Zahorski, Sur la première dérivée, Trans. Amer. Math. Soc. 69 (1950), 1-54.

Department of Mathematics, University of California, Davis, California 95616

Chi Systems, 150 Aero Camino, Goleta, California 93017

Department of Mathematics, University of California, Santa Barbara, California 93106

Department of Mathematics, Michigan State University, East Lansing, Michigan 48824 